\title{
Supersaturated electrolyte solutions: Theory and experiment
}

\author{
Alexander F. Izmailov, Allan S. Myerson, and Han-Soo Na \\ School of Chemical and Materials Science, Polytechnic University, Six MetroTech Center, Brooklyn, New York 11201
}

(Received 2 September 1994; revised manuscript received 17 July 1995)

\begin{abstract}
Highly supersaturated electrolyte solutions can be prepared and studied employing an electrodynamic levitator trap (ELT) technique. The ELT technique involves containerless suspension of a microdroplet thus eliminating dust, dirt, and container walls which normally cause heterogeneous nucleation. This allows very high supersaturations to be achieved. A theoretical study of the experimental results obtained for the water activity in microdroplets of various electrolyte solutions is based on the development of the Cahn-Hilliard formalism for electrolyte solutions. In the approach suggested the metastable state for electrolyte solutions is described in terms of the conserved order parameter $\omega(r, t)$ associated with fluctuations of the mean solute concentration $n_{0}$. Parameters of the corresponding Ginzburg-Landau free energy functional which defines the dynamics of metastable state relaxation are determined and expressed through the experimentally measured quantities. A correspondence of $96-99 \%$ between theory and experiment for all solutions studied was achieved and allowed the determination of an analytical expression for the spinodal concentration $\boldsymbol{n}_{\mathrm{spin}}$ and its calculation for various electrolyte solutions at 298 $K$. The assumption that subcritical solute clusters consist of the electrically neutral Bjerrum pairs has allowed both analytical and numerical investigation of the number-size $N_{c}$ of nucleation monomers (aggregates of the Bjerrum pairs) which are elementary units of the solute critical clusters. This has also allowed estimations for the surface tension $\alpha$, and equilibrium bulk energy $\beta$ per solute molecule in the nucleation monomers. The dependence of these properties on the temperature $T$ and on the solute concentration $n_{0}$ through the entire metastable zone (from saturation concentration $n_{\text {sat }}$ to spinodal $n_{\text {spin }}$ ) is examined. It has been demonstrated that there are the following asymptotics: $N_{c}=1$ at spinodal concentration and $N_{c}=\infty$ at saturation.
\end{abstract}

PACS number(s): 82.60.Nh, 64.60.My, 82.30.Nr, 82.60.Lf

\section{INTRODUCTION}

Let us consider a binary solution consisting of a strong electrolyte and a solvent. Strong electrolytes when dissolved almost completely dissociate into ions. The thermodynamics of these electrolyte solutions are different from the thermodynamics of nonelectrolyte binary solutions. Electrolyte (ionic) solutions possess properties that are absent in solutions composed of neutral particles. Many of these properties are associated in some way with the slow $r^{-1}$ decay of the Coulomb potential and the corresponding $k^{-2}$ singularity in the Fourier transform. The effect of the Coulomb interaction forces between dissolved ions was investigated by Debye-Hückel [1]. They demonstrated that the electrolyte solution energy $E$ acquires a term proportional to the $\frac{3}{2}$ power of the mean solute concentration $n_{0}$. In binary nonelectrolyte solutions all thermodynamic functions can be expanded in integer powers of the concentration $n_{0}$. It is straightforward to conclude that the problem of determining thermodynamic quantities of strong electrolyte solutions reduces to the problem of a completely ionized gas which has been thoroughly discussed in [1] for the case of dilute strong electrolyte solutions.

The physical situation discussed in this paper corresponds to highly supersaturated strong electrolyte solutions. Although the problem is of scientific interest and practical importance, to the best of our knowledge there has been no attempt to provide theoretical insight as well as to develop high-precision measurements.

It is well known [2-4] that a supersaturated solution of a strong electrolyte is in a metastable state. Metastable states are characterized by the permanent birth-death process of subcritical solute clusters which are homogeneously and randomly distributed over the entire solution volume $V$. For these clusters it is energetically favorable to dissolve rather than to grow. This situation lasts until the critical or/and supercritical solute clusters appear. For these clusters it becomes energetically more favorable to grow than to dissolve and nucleation occurs. The time required for a nucleation event to occur is known as the nucleation induction time.

Let us consider an electrolyte solution containing $n_{1}$, $n_{2}, \ldots n_{s}$ ions per unit volume of the various ion species denoted by subscripts $(1,2, \ldots, s)$ which possess the charges $q_{1}, q_{2}, \ldots q_{s}$, respectively. Using this notation the condition of electrical neutrality of the electrolyte solution acquires the form

$$
\sum_{i=1}^{s} n_{i} q_{i}=0
$$

The solvent in this description is treated as a continuous medium, formed from neutral and polar molecules, which is characterized only by its dielectric constant $\varepsilon$. Since the Coulomb forces act between all pairs of ions their motion is not entirely random. As a result, the presence of an ion at a given location in the solution will affect the 
spatial distribution of the other ions in its immediate vicinity. Thus, each ion creates around itself a nonuniformly charged "ion cloud," which on average is spherically symmetrical. In other words, if we select any particular ion, say a $j$ ion, as the center of the solution coordinate system and consider the density distribution of other ions relative to that ion, this density will depend only on the distance $\mathbf{r}$ from the $j$ ion to the ion considered. Thus, let the spatial distribution density $n_{i}(r)$ of the $i$ ion in the ion cloud at the spatial point with the radius vector $\mathbf{r}$ in the $j$-ion-centered coordinate system be defined as follows (the subscript $j$ is not attached to the $i$ ion spatial distribution density $n_{i}(\mathbf{r})$ as well as to the electrostatic potential $\varphi_{i}(\mathbf{r})$ at the $i$-ion location. However, it should be kept in mind that these quantities are only meaningful in the (moving) $j$-ion-centered coordinate system):

$$
n_{i}=\int_{V} d^{3} r n_{i}(r)
$$

The potential-energy density due to the electronic interaction between ions and an electric field produced by all the others ions around them is

$$
U_{0}(\mathbf{r})=-\sum_{i=1}^{s} q_{i} n_{i, 0} \varphi_{i}(\mathbf{r}), \quad q_{i}=e z_{i},
$$

where $z_{i}$ is the $i$ ion charge number and $e$ is the unit positive charge. In this expression $n_{i, 0}$ is the mean $i$-ion concentration in solution and $\varphi_{i}(\mathbf{r})$ is the electrostatic potential at the $i$-ion location produced by all the other ions. The electrostatic interaction between ions gives birth, therefore, to the following correction $E_{\text {corr }}(T, V)$ for the electrolyte solution energy $E(T, V)$ :

$$
\begin{aligned}
& E(T, V)=E_{0}(T, V)+E_{\text {corr }}(T, V), \\
& E_{\text {corr }}(T, V)=\Delta U_{0}(T, V),
\end{aligned}
$$

where

$\Delta U_{0}(T, V)=-\frac{V}{2} \sum_{i=1}^{s} q_{i} n_{i, 0}\left[\varphi_{i}(\mathbf{r})-\varphi_{i, i}(\mathbf{r})\right], \quad r=|\mathbf{r}|$.

The energy $E(T, V)$ is defined for the case when all ions bear their full charges $q_{1}, \ldots, q_{s}$. The energy $E_{0}(T, V)$ is the solution energy when all ions are completely discharged and $T$ is the solution temperature. For convenience, we exclude from the $E_{\text {corr }}(T, V)$ energy expression terms which arise from the ions electrostatic selfenergy. In these terms the potential $\varphi_{i, i}(r)$ is the Coulomb field potential of the $i$ ion itself.

\section{THEORETICAL MODEL FOR SUPERSATURATED ELECTROLYTE SOLUTIONS}

Supersaturated electrolyte solutions are metastable [2-4]. This means that the dissolved solute ions are associated with each other in subcritical solute clusters. These clusters appear due to local fluctuations of the solute concentration $n$. It is energetically favorable for subcritical solute clusters to dissolve rather than to grow. This condition continues until the onset of nucleation, when critical and/or supercritical solute clusters form. For these clusters it is energetically favorable to grow rather than to dissolve. In this way a one-phase metastable state of homogeneous supersaturated solution with the mean solute concentration $n_{0}$ relaxes to a two-phase stable state by forming a heterogeneous saturated solution with the solute concentration $n_{\text {sat }}$. The excess solute concentration, $\Delta n=n_{0}-n_{\text {sat }}$, goes into the creation of solid phase. In this two-phase solution the critical and supercritical solute clusters are identified with the stable solute-rich phase whereas the solution itself is identified with the solute-poor phase. The relaxation process from the one-phase metastable state to the two-phase stable state is known as nucleation. The next stage of the metastable state relaxation is related to the growth of supercritical solute clusters followed by their coalescence.

In this paper we are interested in the nucleation stage of metastable state relaxation in electrolyte solutions. The formalism describing this stage of relaxation is based on the introduction of the local and time-dependent order parameters $\omega_{i}(\mathbf{r}, t)[3-6]$. These order parameters can be defined as the scalar functions:

$$
\omega_{i}(\mathbf{r}, t)=n_{i}(\mathbf{r}, t)-n_{i, 0},
$$

where $n_{i}(r, t)$ is the local current concentration of the $i$ ions. Therefore, the order parameters $\omega_{i}(\mathbf{r}, t)$ describe concentration fluctuations at the spatial-time location $(\mathbf{r}, t)$. These parameters can be introduced for the both phases: the symmetric one, which is associated with the undersaturated homogeneous solutions, and the nonsymmetric one, which is associated with the saturatedsupersaturated solutions. These parameters are random quantities. Therefore, the experimentally observable quantities are their expectation values $\omega_{i}(\mathbf{r})$ :

$$
\omega_{i}(\mathbf{r})=\frac{1}{t_{0}} \int_{0}^{t_{0}} d t \omega_{i}(\mathbf{r}, t),
$$

where $t_{0}$ is the macroscopic observation time (in the metastable state $t_{0}$ is less than the metastable state lifetime). These expectations are equal to zero: $\omega_{i}(\mathbf{r})=0(i=1, \ldots, s)$ for the undersaturated states as well as for the supersaturated states at the vicinity of equilibrium (coexistence or binodal) line. This is understandable since the concentration $n_{i}(r, t)$ fluctuations $\Delta n_{i}(\mathbf{r}, t)$ are negligible in these states and can be of both signs with respect to $n_{i}(r, t)$ with the same probability. Therefore, with good accuracy there exist the equalities $n_{i}(r, t)=n_{i, 0}$. However, the expectations $\omega_{i}(r)$ can be nonzeros: $\omega_{i}(r) \neq 0$ for the supersaturated states at the proximity of spinodal line due to the noticeable and nonnegligible local fluctuations at the vicinity of the region of absolutely unstable states. The theoretical estimation of the order of magnitude of the fluctuations $\Delta n_{i}(r, t)$ at the proximity of spinodal line requires a separate study. However, in order to justify the approximation of the negligible fluctuations $\Delta n_{i}(r, t)$ and, thus, the comparison between theory and experiment presented in this paper we have taken into account only those metastable states in which all the performed measurements had a standard 
deviation within $5 \%$.

Since the total solute concentration $n$ as well as the $i$ ion concentrations $n_{i}$ are conserved quantities, the order parameters $\omega_{i}(r, t)$ are conserved order parameters as well. This means that the following equalities exist:

$$
\frac{\partial}{\partial t}\left[\int_{V} d^{3} r \omega_{i}(\mathbf{r}, t)\right]=0 .
$$

The metastable state in solution is usually created by means of a first-order phase transition, for instance by a quenching procedure [2-4]. The nucleation stage of the metastable state relaxation is described in terms of the order parameters $\omega_{i}(\mathbf{r}, t)$ relaxation. It is noteworthy that one can consider the order parameter $\omega_{i}(\mathbf{r}, t)$ relaxation independently on the relaxation of other thermodynamic quantities only under an assumption that the order parameter $\omega_{i}(\mathbf{r}, t)$ relaxation time $\tau_{i}$ is large enough to guarantee the existence of partial equilibrium for every specific value of the nonequilibrium order parameter. There are two approaches which describe the relaxation of the order parameter $\omega_{i}(r, t)$. They are the GinzburgLandau formalism developed for the case of nonconserved order parameter $[3-6,7]$ and the Cahn-Hilliard formalism developed for the case of conserved order parameter [3-6,8,9]. The main assumption in both approaches is that nucleation can be associated with a Markov's random process. In our case we will make use of the Cahn-Hilliard formalism. Within this formalism the nucleation stage of relaxation is given in terms of the order parameters $\omega_{i}(r, t)$ relaxation equations:

$$
\frac{\partial \omega_{i}(\mathbf{r}, t)}{\partial t}=\Gamma_{i} \nabla^{2} \frac{\partial F(\omega, \varphi ; t)}{\partial \omega_{i}(\mathbf{r}, t)}, i=1, \ldots, s,
$$

where

$$
F(\omega, \varphi ; t)=\int_{V} d^{3} r F(\omega, \varphi ; \mathrm{r}, t) .
$$

In the above relaxation equations $\Gamma_{i}$ is the $i$-ion mobility coefficient and $F(\omega, \varphi ; t)$ is the time-dependent Helmholtz free-energy functional for the supersaturated electrolyte solution. This functional describes the free energy of the order parameter $\omega$ subsystem and the ion cloud $\varphi$ subsystem which interact with each other at the given solution temperature and volume. Therefore, the functional $F(\omega, \varphi ; t)$ density $F(\omega, \varphi ; r, t)$ should contain the following terms:

$F(\omega, \varphi ; \mathrm{r}, t)=F(\omega ; \mathrm{r}, t)+F(\varphi ; \mathrm{r}, t)+F_{\mathrm{int}}(\omega, \varphi ; \mathrm{r}, t)$.

In expression (5) the Helmholtz free-energy density functional $F(\omega ; \mathbf{r}, t)$ of the metastable $\omega$ subsystem can be presented as the following Landau expansion with respect to even powers of the order parameters $\omega_{i}(r, t)$ :

$$
\begin{aligned}
& F(\omega ; \mathbf{r}, t)=\sum_{i=1}^{s} \mid \frac{B_{i}}{2} \frac{\left[\nabla \omega_{i}(\mathbf{r}, t)\right]^{2}}{n_{i, 0}}+\frac{\mu_{i}}{2} \frac{\omega_{i}^{2}(\mathbf{r}, t)}{n_{i, 0}} \\
& \left.+\frac{\eta_{i}}{4} \frac{\omega_{i}^{4}(\mathbf{r}, t)}{n_{i, 0}^{3}}\right) \text {, }
\end{aligned}
$$

where the parameters $B_{i}$ are proportional to the square of the correlation radii $r_{c, i}$ [5]. These radii determine the magnitude of distances at which the correlations of the order parameters $\omega_{i}(\mathbf{r}, t)$ fluctuations decrease significantly. The parameters $\mu_{i}$ and $\eta_{i}$ determine boundaries of the $\omega$-subsystem metastable zone. Since the functional $F(\omega ; \mathrm{r}, t)$ does not contain interaction terms between the various order parameters $\omega_{i}(r, t)$ $(i=1, \ldots, s)$ it is assumed that concentration fluctuations of the ions of different kinds are independent. Therefore, the functional $F(\omega ; \mathrm{r}, t)$ represents the simplest form for the $\omega$-subsystem Helmholtz free-energy density which allows existence of metastability.

The $\varphi$-subsystem Helmholtz free-energy density functional $F(\varphi ; \mathbf{r}, t)$ has the form

$$
\begin{aligned}
& F(\varphi ; \mathbf{r}, t)=\frac{1}{2 c^{2}}\left[\frac{\partial \varphi(\mathbf{r}, t)}{\partial t}\right]^{2}+U(\varphi ; \mathbf{r}, t), \\
& U(\varphi ; \mathbf{r}, t)=\frac{1}{2}[\nabla \varphi(\mathbf{r}, t)]^{2}
\end{aligned}
$$

where $c$ is the light speed in a solution. In this expression the term $U(\varphi ; r, t)$ is the $\varphi$-subsystem potential-energy density. It is assumed that (i) the $\varphi$ subsystem, which is described in terms of the scalar field $\varphi(r, t)$ is always in equilibrium, and (ii) the $\varphi$-subsystem equilibrium state cannot be destroyed even by interaction with the $\omega$ subsystem.

The interaction term $F_{\text {int }}(\omega, \varphi ; \mathrm{r}, t)$ between the $\omega$ and $\varphi$ subsystems has the form

$$
F_{\mathrm{int}}(\omega, \varphi ; \mathbf{r}, t)=-\frac{e}{\varepsilon_{0} \varepsilon} \varphi(\mathbf{r}, t) \sum_{i=1}^{s} z_{i} \omega_{i}(\mathbf{r}, t),
$$

where $\varepsilon_{0}$ is the vacuum permittivity constant. Expression (8) represents simple electrostatic interaction between the ion-cloud potential $\varphi(r, t)$ due to all kinds of ions and an electric charge at the spatial-time point $(r, t)$.

To complete the description of the interacting $\omega$ and $\varphi$ subsystems one has to supplement Eq. (4), describing the evolution of the parameters $\omega_{i}(r, t)$, with a similar equation for the ion-cloud potential $\varphi(r, t)$. This can be done by means of the Euler-Lagrange equation:

$$
\frac{\partial}{\partial t}\left[\frac{\delta A(\omega, \varphi ; t)}{\delta[\partial \varphi(\mathrm{r}, t) / \partial t]}\right]+\nabla \frac{\delta A(\omega, \varphi ; t)}{\delta \nabla \varphi(\mathrm{r}, t)}=\frac{\delta A(\omega, \varphi ; t)}{\delta \varphi(\mathrm{r}, t)},
$$

where $A(\omega, \varphi ; t)$ is the action functional for the supersaturated electrolyte solution. An expression for this functional in terms of the notations introduced above is straightforward:

$$
\begin{aligned}
A(\omega, \varphi ; t)= & \int_{V} d^{3} r A(\omega, \varphi ; \mathrm{r}, t) \\
A(\omega, \varphi ; \mathrm{r}, t)= & \frac{1}{2 c^{2}}\left(\frac{\partial \varphi(\mathrm{r}, t)}{\partial t}\right)^{2}-U(\varphi ; \mathrm{r}, t) \\
& -F(\omega ; \mathrm{r}, t)-F_{\mathrm{int}}(\omega, \varphi ; \mathrm{r}, t)
\end{aligned}
$$

One can rewrite Eqs. (4) and (9) as follows: 


$$
\begin{aligned}
& \frac{\partial \omega_{i}(\mathbf{r}, t)}{\partial t}=\Gamma_{i} \nabla^{2}[-\frac{B_{i}}{n_{i, 0}} \nabla^{2} \omega_{i}(\mathbf{r}, t)-\frac{e}{\varepsilon_{0} \varepsilon} z_{i} \varphi(\mathbf{r}, t) \\
&\left.+\mu_{i} \frac{\omega_{i}(\mathbf{r}, t)}{n_{i, 0}}+\eta_{i}\left[\frac{\omega_{i}(\mathbf{r}, t)}{n_{i, 0}}\right]^{3}\right], \\
& \frac{1}{2 c^{2}} \frac{\partial^{2} \varphi(\mathbf{r}, t)}{\partial t^{2}}-\nabla^{2} \varphi(\mathbf{r}, t)=\frac{e}{\varepsilon_{0} \varepsilon} \sum_{i=1}^{s} z_{i} \omega_{i}(\mathbf{r}, t) .
\end{aligned}
$$

Let us consider the joint solution of Eqs. (11a) and (11b) in the stationary limit. This limit corresponds to the establishment of partial equilibrium between solution and subcritical ionic clusters. Characteristic times $\tau_{i}$ $(i=1,2, \ldots, s)$ needed to establish the partial equilibrium should be much less than the induction time. Thus, in the stationary limit, i.e., under the conditions that $\partial \omega_{i}(\mathbf{r}, t) / \partial t=0$ and $\partial \varphi(\mathbf{r}, t) / \partial t=0$, the spherically symmetrical solutions $\varphi(r)$ and $\omega_{i}(r)$ satisfy the following equations:

$$
\begin{aligned}
& -\frac{B_{i}}{n_{i, 0}} \nabla^{2} \omega_{i}(r)-\frac{e}{\varepsilon_{0} \varepsilon} z_{i} \varphi(r)+\mu_{i} \frac{\omega_{i}(r)}{n_{i, 0}} \\
& +\eta_{i}\left|\frac{\omega_{i}(r)}{n_{i, 0}}\right|^{3}=0, \\
& \nabla^{2} \varphi(r)=-\frac{e}{\varepsilon_{0} \varepsilon} \sum_{i=1}^{s} z_{i} \omega_{i}(r) .
\end{aligned}
$$

It is noteworthy that the last equation coincides with the electrostatic Poisson equation [1].

The properties of the $\omega$ subsystem which are described in terms of the Landau time-dependent Helmholtz freeenergy density functional $F(\omega ; \mathbf{r}, t)$ are well known near the critical point $[5,7]$. There is a critical point if and only if the coefficients $\mu_{i}=-\left|\mu_{i}\right|$ are less than zero $\left(\mu_{i}<0\right)$. As it follows from the general order-parameter formalism $[5,7]$, the square of the correlation radii $r_{c, i}$ can be defined in the form $r_{c, i}^{2}=B_{i} /\left(2\left|\mu_{i}\right|\right)$. Therefore, the term $\left(B_{i} / \mu_{i}\right) \nabla^{2} \omega(r)$ is proportional to the ratio $\left(r_{c} / l_{i}\right)^{2}$, where the length $l_{i}$ characterizes the distance at which the field $\omega_{i}(r)$ changes significantly. In order to evaluate the ratio $r_{c} / l_{i}$ let us assume that the following conditions are satisfied.

(i) The metastable state is far from a critical point, i.e., $r_{c} \ll \infty$. This is usually the case for electrolyte solutions of lower molecular weight solutes. In such solutions the critical point has never been observed under normal pressure.

(ii) The critical radius of nucleation $\boldsymbol{R}_{c}$ is much greater than the correlation radii $r_{c, i}\left(\boldsymbol{R}_{c} \gg r_{c, i}\right)$. The opposite case when $R_{c} \approx r_{c, i}$ corresponds to the unlikely situation of immediate nucleation (zero induction time) after the solution enters the metastable state.

Under these conditions the ratio $r_{c, i} / l_{i}$ is small: $r_{c, i} / l_{i} \ll 1$, since $R_{c} \sim l_{i}$. Therefore, Eq. (12a) can be simplified as follows:

$$
\begin{gathered}
\omega_{i}(r)+\frac{\eta_{i}}{\mu_{i}} \frac{\omega_{i}^{3}(r)}{n_{i, 0}^{2}}=F_{i}(r), \\
F_{i}(r)=\frac{e}{\varepsilon_{0} \varepsilon \mu_{i}} z_{i} n_{i, 0} \varphi(r) .
\end{gathered}
$$

Let us seek for solution of Eq. (13a) in the following form:

$$
\omega_{i}(r)=\sum_{m=0}^{\infty}\left[\frac{\eta_{i}}{2 \mu_{i}}\right)^{m} \omega_{i, m}(r) .
$$

The ratios $\mu_{i} / \eta_{i}$ determine the equilibrium values $\omega_{i, \text { eq }}$ of the order parameters $\omega_{i}(r): \omega_{i, \text { eq }} / n_{i, 0}=\left(\mu_{i} / \eta_{i}\right)^{1 / 2}$, where $i=1, \ldots, s$. The Landau order-parameter formalism for the Helmholtz free energy is correct only if the Ginzburg-Levanyuk criterion $[10,11]$ is satisfied. This criterion requires that the equilibrium values $\omega_{i, \text { eq }}$ of the order parameters $\omega_{i}(r)$ must be much greater than their standard deviations:

$$
\begin{aligned}
\frac{\mu_{i}}{\eta_{i}} & \gg \frac{1}{n_{i, 0}^{2}}\left\langle\Delta \omega_{i}\left(r_{1}\right) \Delta \omega_{i}\left(r_{2}\right)\right\rangle \\
& =\frac{\mu_{i}}{\eta_{i}}\left(\frac{\left\langle\omega_{i}\left(r_{1}\right) \omega_{i}\left(r_{2}\right)\right\rangle}{\omega_{i, \mathrm{eq}}^{2}}-1\right) .
\end{aligned}
$$

From the above criterion it follows that $\left|\eta_{i} / 2 \mu_{i}\right| \ll<$. Therefore, considering expansion (14a) it is natural to keep only the first two terms which can be found in the following form:

$$
\omega_{i, 0}(r)=F_{i}(r), \quad \omega_{i, 1}(r)=-\frac{F_{i}^{3}(r)}{2 n_{i, 0}^{2}} .
$$

The next step is to substitute solution for the order parameters $\omega_{i}(r)$ given by expressions (14a) and (15) into the Poisson equation (12b). This substitution leads to the following second-order nonlinear ordinary differential equation for the scalar field $\varphi(r)$ :

$$
\nabla^{2} \varphi(r) \approx \kappa_{1}^{2} \varphi(r)+\kappa_{2} \varphi^{3}(r),
$$

where

$$
\begin{aligned}
& \kappa_{1}^{2}=-\left(\frac{e}{\varepsilon_{0} \varepsilon}\right)^{2} \sum_{i=1}^{s} \frac{z_{i}^{2}}{\mu_{i}} n_{i, 0}, \\
& \kappa_{2}=\left(\frac{e}{\varepsilon_{0} \varepsilon}\right)^{4} \sum_{i=1}^{s}\left[\frac{z_{i}}{\mu_{i}}\right]^{4} \eta_{i} n_{i, 0} .
\end{aligned}
$$

It is noteworthy that under the condition $\kappa_{2} \ll \kappa_{1}^{2}$ one can reduce the approach developed for supersaturated electrolyte solutions to the limiting case corresponding to undersaturated electrolyte solutions. Analytical solution for this limiting case was given by Debye and Hückel [1]. In order to obtain the complete correspondence between the reduced $\left(\kappa_{2} \ll<\kappa_{1}^{2}\right)$ equation $\left(13^{\prime}\right)$ and the Debye-Hückel result for the equation determining the ion-cloud potential $\varphi(r)$ in undersaturated electrolyte solutions one has to specify the parameters $\mu_{i}$ of the Landau free-energy functional (6) as follows:

$$
\mu_{i}=\mu=-|\mu|, \quad|\mu|=k T .
$$

Thus, this specification has allowed us to determine parameters $\mu_{i}$ of the Landau free-energy functional in the temperature region remote from the critical point. Therefore, in order to reduce Eq. $\left(13^{\prime}\right)$ describing the case corresponding to saturated-supersaturated electro- 
lyte solutions to undersaturated electrolyte solutions it is necessary to impose the following constraints on the Ginzburg-Landau parameters $\mu_{i}$ and $\eta_{i}$ : (i) the parameters $\mu_{i}$ are the same for all kinds of ions and are equal to $-k T$, and (ii) the parameters $\eta_{i}$ are all equal to zero $\left(\eta_{i}=0\right)$. These constraints are very natural since they eliminate the nonlinear terms from the Helmholtz freeenergy density function $F(\omega ; \mathrm{r}, t)$ [see expression (6)] reducing it to the expression corresponding exactly to the Helmholtz free-energy density function of the undersaturated electrolyte solutions [1]. Thus, in terms of Eq. $\left(13^{\prime}\right)$ the effect of the electrolyte solution metastability is given by the non-linear term proportional to $\varphi^{3}(r)$.

Let us seek for solution of Eq. (13') at the $i$-ion location in the following form:

$$
\varphi(r)=\varphi_{i}(r)=\sum_{m=0}^{\infty}\left(e^{2} \kappa_{2}\right)^{m} \varphi_{i, m}(r),
$$

where $e^{2} \kappa_{2}$ is the dimensionless parameter. Substitution of this expansion into Eq. (13') allows us to find its first terms in the form

$$
\begin{aligned}
& \varphi_{i, 0}(r)=e\left[\frac{z_{i} A}{4 \pi \varepsilon_{0} \varepsilon}\right) \frac{e^{-\kappa_{1} r}}{r}, A=\frac{e^{\kappa_{1} a}}{1+\kappa_{1} a}, \\
& \varphi_{i, 1}(r)=-e\left(\frac{z_{i} A}{4 \pi \varepsilon_{0} \varepsilon}\right)^{3} \frac{E_{2}\left(2 \kappa_{1} a\right)}{2 \kappa_{1} a} \frac{e^{-\kappa_{1} r}}{r}, \\
& E_{2}(z)=\int_{1}^{\infty} d t \frac{e^{-z t}}{t^{2}},
\end{aligned}
$$

where $a$ is the minimum average distance to which ions, both positive and negative, can approach one another. Assuming now that the constant $e^{2} \kappa_{2}$ is much less than unit $^{2}\left(e^{2} \kappa_{2} \ll 1\right)$ (This assumption will be verified in Sec. VII) one can present the result for the potential $\varphi_{i}(r)$ of the supersaturated electrolyte solution as follows:

$$
\begin{aligned}
\varphi_{i}(r)= & e\left(\frac{z_{i} A}{4 \pi \varepsilon_{0} \varepsilon}\right)\left[1-e^{2} \kappa_{2}\left(\frac{z_{i} A}{4 \pi \varepsilon_{0} \varepsilon}\right)^{2} \frac{E_{2}\left(2 \kappa_{1} a\right)}{2 \kappa_{1} a}\right] \frac{e^{-\kappa_{1} r}}{r} \\
& +o\left[\left(e^{2} \kappa_{2}\right)^{2}\right] .
\end{aligned}
$$

It is noteworthy that within the approach developed for supersaturated electrolyte solutions the effect of solution metastability leads to simple renormalization of the potential $\varphi_{i}(r)$ constant leaving the potential $\varphi_{i}(r)$ functional dependence on $r$ the same as it is for the undersaturated electrolyte solutions.

\section{THERMODYNAMICS OF THE SUPERSATURATED ELECTROLYTE SOLUTIONS}

Let us return to expression (3) for the solution energy $E(T, V)$. It follows from the previous considerations that (i) $E_{0}(T, V)$ can be identified with the energy of the undersaturated electrolyte solution consisting of discharged ions, and (ii) the energy $E(T, V)$ corrective term $E_{\text {corr }}(T, V)$ describes the combined effect due to the electrostatic interaction between the charged solute ions and the solution supersaturation. Thus, substituting into expression (3) result (16) for the potential $\varphi_{i}(r)$ it is straightforward to obtain the following expression for $E_{\text {corr }}(T, V)$ :

$$
\begin{aligned}
E_{\text {corr }}(T, V)= & \frac{V}{2} e^{2} \kappa_{1}\left(\frac{A}{4 \pi \varepsilon_{0} \varepsilon}\right) \sum_{i=1}^{s} z_{i}^{2} n_{i, 0} \\
& -\frac{V}{2} e^{4} \kappa_{2}\left(\frac{A}{4 \pi \varepsilon_{0} \varepsilon}\right)^{3} \frac{E_{2}\left(2 \kappa_{1} a\right)}{2 a} \sum_{i=1}^{s} z_{i}^{4} n_{i, 0}
\end{aligned}
$$

where the temperature-dependent expressions for $\kappa_{1}$ and $\kappa_{2}$ are given by relations $\left(13^{\prime}\right)$.

The Cahn-Hilliard formalism introduced in Sec. II implies that partial thermodynamic equilibrium exists in a metastable state. For the case of nucleation, the lifetime of this partial equilibrium can be associated with the induction time. Therefore, within the induction time all equalities of equilibrium thermodynamics exist including the relationship between the solution energy corrective term $E_{\text {corr }}(T, V)$ and its Helmholtz free energy $F(T, V)$ :

$$
\frac{E_{\text {corr }}(T, V)}{T^{2}}=-\frac{\partial}{\partial T}\left(\frac{F(T, V)-F_{0}(T, V)}{T}\right)_{V} .
$$

In this expression $F_{0}(T, V)$ is the Helmholtz free energy of undersaturated electrolyte solution consisting of discharged ions:

$$
F_{0}(T, V)=\mathcal{C}(T, V)+V \sum_{i=1}^{s}\left[k T n_{i, 0} \ln \left(n_{i, 0}\right)+n_{i, 0} \Psi_{i}(T, V)\right],
$$

where $\mathcal{C}(T, V)$ and $\Psi_{i}(T, V)(i=1, \ldots, s)$ are some constants at the given temperature and volume. Integration of relationship (18) gives the following result for the Helmholtz free energy $F(T, V)$ :

$$
\begin{aligned}
F(T, V)= & \mathcal{C}(T, V)+V \sum_{i=1}^{s}\left[k T n_{i, 0} \ln \left(n_{i, 0}\right)+\Psi_{i}(T, V) n_{i, 0}\right] \\
& +\frac{V e^{3} A}{12 \pi(k T)^{1 / 2}\left(\varepsilon_{0} \varepsilon\right)^{2}}\left[\sum_{i=1}^{s} z_{i}^{2} n_{i, 0}\right]^{3 / 2}-\frac{V e^{8} A^{3} E_{2}\left(2 \kappa_{1} a\right)}{1280 \pi^{3}(k T)^{4}\left(\varepsilon_{0} \varepsilon\right)^{7} a}\left[\sum_{i=1}^{s} z_{i}^{4} n_{i, 0} \eta_{i}\right]\left[\sum_{i=1}^{s} z_{i}^{4} n_{i, 0}\right] .
\end{aligned}
$$

This expression can be simplified by taking into account that the distance $a$ is usually much less than the length $1 / \kappa_{1}\left(a<<1 / \kappa_{1}\right)$ which characterizes the dimension of the ion cloud of a given ion. Therefore, since $a \kappa_{1} \ll 1$ there exist the approximations

$$
E_{2}\left(2 \kappa_{1} a\right) \approx 1, A \approx 1 .
$$

Using these approximations, the expression for the $i$-ion 
chemical potential derivative $d \mu_{i}(T) / d n_{i, 0}$ in the supersaturated electrolyte solutions acquires the form

$$
\begin{aligned}
\frac{d \mu_{i}(T)}{d n_{i, 0}}= & \frac{1}{V} \frac{\partial^{2} F(T, V)}{\partial n_{i, 0}^{2}} \\
= & \frac{k T}{n_{i, 0}}+\frac{z_{i}^{4} e^{3}}{16 \pi(k T)^{1 / 2}\left(\varepsilon_{0} \varepsilon\right)^{2}}\left(\sum_{i=1}^{s} z_{i}^{2} n_{i, 0}\right)^{-1 / 2} \\
& -\frac{\left(z_{i} e\right)^{8} \eta_{i}}{640 \pi^{3}(k T)^{4}\left(\varepsilon_{0} \varepsilon\right)^{7} a} .
\end{aligned}
$$

\section{SPINODAL CONCENTRATION FOR THE CASE OF BINARY SYMMETRICAL ELECTROLYTE SOLUTIONS}

Let us consider the case of binary $(i=1,2)$ symmetrical $\left(z_{1}=-z_{2}=|z|\right)$ electrolyte solutions where the equality $n_{1,0}=n_{2,0}=n_{0}$ is true. In this case the Gibbs-Duhem relationship (which is valid at the states of complete and partial equilibriums) acquires the form

$$
N \frac{d \mu_{\mathrm{siv}}\left(T, n_{0}\right)}{d n_{0}}=-\frac{n_{0}}{2}\left[\frac{d \mu_{1}\left(T, n_{0}\right)}{d n_{0}}+\frac{d \mu_{2}\left(T, n_{0}\right)}{d n_{0}}\right)
$$

where $\mu_{\text {siv }}\left(T, n_{0}\right)$ is the solvent chemical potential and $N$ is the number of solvent molecules per unit volume.
Analyzing expression (20) for the binary symmetrical undersaturated electrolyte solutions $\left(\eta_{1}=\eta_{2}=0\right)$ leads to the following equality:

$$
\frac{d \mu_{1}(T)}{d n_{1,0}}=\frac{d \mu_{2}(T)}{d n_{2,0}}=\frac{d \mu_{\mathrm{slt}}\left(T, n_{0}\right)}{d n_{0}},
$$

where $\mu_{\text {slt }}\left(T, n_{0}\right)$ is the mean solute chemical potential. In order to keep this equality for supersaturated electrolyte solutions one has to require that the parameters $\eta_{1}$ and $\eta_{2}$ of the $\omega$-subsystem density functional (6) have to be the same $\eta_{1}=\eta_{2}=\eta$ for both kinds of ions. Under this requirement the Gibbs-Duhem relationship for the symmetrical supersaturated electrolyte solutions acquires the form

$$
N \frac{d \mu_{\mathrm{stv}}\left(T, n_{0}\right)}{d n_{0}}=-n_{0} \frac{d u_{\mathrm{slt}}\left(T, n_{0}\right)}{d n_{0}},
$$

where

$$
\begin{aligned}
\frac{d \mu_{\mathrm{slt}}\left(T, n_{0}\right)}{d n_{0}}= & \frac{k T}{n_{0}}+\left(\frac{(|z| e)^{3}}{16 \pi(2 k T)^{1 / 2}\left(\varepsilon_{0} \varepsilon\right)^{2}}\right) n_{0}^{-1 / 2} \\
& -\frac{(|z| e)^{8} \eta}{640 \pi^{3}(k T)^{4}\left(\varepsilon_{0} \varepsilon\right)^{7} a} .
\end{aligned}
$$

In this paper we are interested in the solvent activity $\lambda_{\text {slv }}\left(T, n_{0}\right)$ for which there exists the following expression:

$$
\lambda_{\text {slv }}\left(T, n_{0}\right)=\lambda_{\text {slv }}(T, 0) \exp \left(-\frac{1}{k T N} \int_{0}^{n_{0}} d n^{\prime} n^{\prime} \frac{d \mu_{\text {slt }}\left(T, n^{\prime}\right)}{d n^{\prime}}\right),
$$

where $\lambda_{\text {slv }}(T, 0)$ is the activity of pure solvent. Carrying out integration in expression (22a) we obtain the following relation for the activity $\lambda_{\text {slv }}\left(T, n_{0}\right)$ :

$$
\lambda_{\text {slv }}\left(T, n_{0}\right)=\lambda_{\text {slv }}(T, 0) \exp \left[-\frac{1}{N}\left[n_{0}+\frac{2^{1 / 2}(|z| e)^{3}}{48 \pi(k T)^{3 / 2}\left(\varepsilon_{0} \varepsilon\right)^{2}} n_{0}^{3 / 2}-\frac{(z \mid e)^{8} \eta}{1280 \pi^{3}(k T)^{5}\left(\varepsilon_{0} \varepsilon\right)^{7} a} n_{0}^{2}\right]\right] .
$$

The contribution due to the electrolyte solution metastability comes in the form of the third term in the exponent of expression (22b).

It is well understood [3-6] that at the concentration $n_{0}=n_{\text {spin }}(T)$ corresponding to the spinodal line (line which separates metastable and unstable states) at the given temperature $T$ is where the first derivative $d \mu_{\text {stt }}\left(T, n_{0}\right) / d n_{0}$ of the solute chemical potential $\mu_{\text {slt }}\left(T, n_{0}\right)$ with respect to the solute concentration $n_{0}$ becomes equal to zero:

$$
\left.\frac{d \mu_{\text {slt }}\left(T, n_{0}\right)}{d n_{0}}\right|_{n_{0}=n_{\text {spin }}(T)}=0 .
$$

Substitution into this equation of result (21b) for $d \mu_{\text {slt }}\left(T, n_{0}\right) / d n_{0}$ allows derivation of the following equation for the spinodal concentration $n_{\text {spin }}(T)$ :

$$
\begin{aligned}
k T+\frac{(|z| e)^{3}}{16 \pi(2 k T)^{1 / 2}\left(\varepsilon_{0} \varepsilon\right)^{2}} n_{\text {spin }}^{1 / 2}(T) & \\
& -\frac{(|z| e)^{8} \eta}{640 \pi^{3}(k T)^{4}\left(\varepsilon_{0} \varepsilon\right)^{7} a} n_{\text {spin }}(T)=0 .
\end{aligned}
$$

Solution of this simple square equation with respect to $n_{\text {spin }}^{1 / 2}(T)$ and its numerical estimations for various electrolyte solutions will be given in Sec. VII.

\section{SUBCRITICAL SOLUTE CLUSTERS, THEIR ASSOCIATION DEGREE, AND NUCLEATION MONOMERS}

In the simplest model of electrolyte solutions the dissolved ions can be considered as rigid unpolarized spheres contained in a medium of the fixed macroscopic dielectric constant $\varepsilon$. In this description the nonpolar quantum bonds between ions as well as the ion-solvent in- 
teractions are neglected. Within such a model Bjerrum has assumed that two ions of the opposite electric sign at the distance $r<r_{\text {ass }}=(|z| e)^{2} /\left(2 \varepsilon_{0} \varepsilon k T\right)$ are associated [12], i.e., from the Bejerrum electrically neutral pair. If the ions are of like sign the probability of their association is very small.

The solution metastable state is characterized by the appearance of subcritical solute clusters. Let us assume that these clusters are associations of the electrically neutral Bjerrum pairs. In general, such associations, being electrically neutral, may possess dipole moments. However, in this paper it is assumed that the dipole moment of a subcritical solute cluster, averaged over its lifetime, is zero due to the translational and rotational diffusions of these clusters. Thus, an interaction between the cluster dipole moment and the gradient of the ion-cloud potential, averaged over the cluster lifetime, can be neglected. Therefore, within the suggested model the subcritical solute clusters can be treated as electrically neutral with zero dipole moments associations of the Bjerrum pairs. For such clusters it is natural to assume that their associated ions do not contribute the correction energy term $E_{\text {corr }}(T, V)$ for saturated and supersaturated electrolyte solutions [see expression (3)]. Thus, relation (17) for the case of saturated and/or supersaturated solutions can be presented in the following simple Debye-Hückel form:

$$
\begin{aligned}
& E_{\text {corr }}(T, V)=\frac{V}{2} e^{2} \kappa_{1}^{*}\left|\frac{1}{4 \pi \varepsilon_{0} \varepsilon}\right| \sum_{i=1}^{s} z_{i}^{2} n_{i, 0}^{*}, \\
& \kappa_{1}^{*}=\frac{e}{\varepsilon_{0} \varepsilon(k T)^{1 / 2}}\left(\sum_{i=1}^{s} z_{i}^{2} n_{i, 0}^{*}\right]^{1 / 2},
\end{aligned}
$$

where $n_{i, 0}^{*}$ is the number of $i$ ions which are nonassociated into the Bjerrum pairs.

Let us consider as in the preceding section the case of binary symmetrical electrolyte solutions $\left(n_{1,0}=n_{2,0}=n_{0}\right.$, $\eta_{1}=\eta_{2}=\eta$ ). Equating in this case expressions (17) and (24) for the solution energy $E(T, V)$ corrective term $E_{\text {corr }}(T, V)$ it is straightforward to find $n_{0}^{*}$ as the function of the solution temperature $T$ and the mean solute concentration $n_{0}$ :

$$
\begin{aligned}
n_{0}^{*} & =n_{0}^{*}\left(T, n_{0}\right) \\
& =n_{0}\left[1-\frac{n_{0}^{1 / 2} \eta}{16 \sqrt{2} \pi^{2}(k T)^{7 / 2} a}\left[\frac{|z| e}{\varepsilon_{0} \varepsilon}\right]^{5}\right]^{2 / 3} .
\end{aligned}
$$

Therefore, the number $n_{\mathrm{cl}}\left(T, n_{0}\right)$ of ions associated in the Bjerrum pairs can be determined by the following equation:

$$
\begin{aligned}
d\left(T, n_{0}\right) & =\frac{n_{\mathrm{cl}}^{*}\left(T, n_{0}\right)}{n_{0}}=1-\frac{n_{0}^{*}(T)}{n_{0}} \\
& =1-\left[1-\frac{n_{0}^{1 / 2} \eta}{16 \sqrt{2} \pi^{2}(k T)^{7 / 2} a}\left[\frac{|z| e}{\varepsilon_{0} \varepsilon}\right]^{5}\right]^{2 / 3} .
\end{aligned}
$$

The ion association degree introduced in this equation, $d\left(T, n_{0}\right)$ determines the probability that an arbitrary ion belongs to the Bjerrum pair. This probability has the local maximum equal to unit $\left[d\left(T, n_{0}\right)=1\right]$ at the solute concentration equal to $n_{0, \max }(T)$ :

$$
n_{0, \max }(T)=512 \pi^{4}(k T)^{7}\left(\frac{\varepsilon_{0} \varepsilon}{z e}\right)^{10}\left(\frac{a}{\eta}\right)^{2} .
$$

The temperature-dependent solute concentration $n_{0, \max }(T)$ corresponds to such a metastable state when all ions are associated into the Bjerrum pairs. This means that at the concentration $n_{0, \max }(T)$ the electrolyte solution converts into a nonelectrolyte solution since all the ions become associated in the electrically neutral Bjerrum pairs. As the solute concentration $n_{0}$ is increased further, single ions appear until the spinodal concentration $n_{\text {spin }}(T)$ is reached.

In the classical approach to statistical mechanics of clusters [13] it is assumed that the Helmholtz free energy $F_{l}\left(T, n_{0}\right)$ of the cluster containing $l$ particles (in our case containing $l$ Bejerrum's pairs) has the following form:

$$
F_{l}\left(T, n_{0}\right)=k T\left[\alpha(T) l^{2 / 3}+\beta(T) l-\ln \left(n_{0}\right) l\right],
$$

where $n_{0}$ is the mean concentration of solute molecules, $\alpha(T)$ is proportional to the surface tension, and $\beta(T)$ is the equilibrium bulk energy per solute molecule in the solute-rich phase (in clusters). Therefore, expression for the Helmholtz free energy describes subcritical solute clusters as an open (grand) canonical ensemble of the Bjerrum pair associations where only the average, rather than the exact number of the associated pairs, is specified. This means that the solute clusters, the Bjerrum pair associations, can exchange the pairs between themselves as well as with the solute-poor phase (solution). In such a treatment of the Helmholtz free energy the term $k T \ln \left(n_{0}\right)$ corresponds to the chemical potential $[4,5]$ of a single Bjerrum's pair in the solution. The grand partition function $\Xi\left(T, n_{0}\right)$ of the ensemble of subcritical solute clusters is equal, by definition, to the ion association degree $d\left(T, n_{0}\right)$ and can be presented as follows:

$$
\Xi\left(T, n_{0}\right)=d\left(T, n_{0}\right)=\mathrm{const} \times \sum_{l=1}^{N_{c}\left(T, n_{0}\right)} e^{-F_{1}\left(T, n_{0}\right) / k T},
$$

where const is the normalization constant and $N_{c}\left(T, n_{0}\right)$ is the number size of nucleation monomer which is temperature and solute concentration dependent. In expression (29) we account only for the noninteracting nucleation monomers (elementary solute units appearing in the result of nucleation), aggregations of which constitute critical solute clusters. When the number of the Bjerrum pairs associated in any subcritical solute cluster reaches $N_{c}\left(T, n_{0}\right)$, the nucleation onset begins and nucleation monomers form. Equation (29) represent a classical point of view for the nucleation of subcritical solute clusters $[2-6,13]$. This equation being combined with result $(26)$ for the association degree $d\left(T, n_{0}\right)$, obtained within the nonclassical Ginzburg-Landau formalism [3-7], allows determination of such classical concepts as the surface tension $\alpha(T)$ and the equilibrium bulk energy $\beta(T)$ as 
well as the number size $N_{c}\left(T, n_{0}\right)$ of nucleation monomers. Estimation of these concepts will be given in Sec. VII.

\section{EXPERIMENTAL STUDY OF SUPERSATURATED ELECTROLYTE SOLUTIONS}

In order to experimentally investigate properties of highly supersaturated solutions it is necessary to eliminate heterogeneous nucleation. For this purpose the electrodynamic levitation technique has been developed [14-16] in order to measure the microdroplet solvent activity $\lambda_{\text {svv }}\left(T, n_{0}\right)$ as a function of the solution temperature $T$ and the mean solute concentration $n_{0}$. In this technique a micrometer-sized solution droplet is produced by the particle generator and after being electrically charged it is injected inside the electrodynamic levitator trap (ELT), which is incased in a vacuum chamber. The ELT consists of three electrodes. In our experiments performed with the spherical void electromagnetic levitator trap (SVELT) [17-21], the top and bottom electrodes are spheriods of revolution and the center electrode is torus having a spherical cross section. The time-varying voltage $V_{\mathrm{ac}}(t)$ is applied to the torus and, relative to the top and bottom electrodes interior, a nearly perfect oscillating quadrupole potential is produced. This potential, even being quite moderate, forces the electrically charged solution microdroplet to oscillate vertically along the SVELT axis and below its geometric center. Introduction of the constant voltage $V_{\mathrm{dc}}$, which is divided equally between the top and center, and the center and bottom electrodes produce a static interior potential. This potential balances gravity at the SVELT null point and, thus, allows the microdroplet to be brought to the SVELT geometrical center where the ac potential $V_{\mathrm{ac}}(t) \sim \cos (2 \pi v t)$ is zero and the microdroplet comes to rest. A diagram of the experimental setup after the solution microdroplet being injected is shown schematically in Fig. 1. Thus, since the suspended solution microdroplet is containerless (free of foreign surface) the very high supersaturations can be achieved before the nucleation onset starts. In this way Rubel [14] measured the water activity at supersaturations as a function of solute concentration for aqueous phosphoric acid solutions using a bihyperboloidal electrodynamic balance trap in a continuously flowing system. Using almost the same experimental setup, Tang and co-workers [15] measured the water activity of the supersaturated aqueous electrolyte solutions of sodium chloride $\mathrm{NaCl}$ and potassium chloride KCl. Richardson and Spann [16] used the ELT technique to study the water activity of aqueous solutions of ammonium sulfate $\left(\mathrm{NH}_{4}\right)_{2} \mathrm{SO}_{4}$. In the present study we have investigated water activity of the highly supersaturated aqueous electrolyte solutions of sodium bromide $(\mathrm{NaBr})$, potassium chloride and bromide $(\mathrm{KCl}$ and $\mathrm{KBr}$ ), and ammonium chloride and sulfate $\left[\mathrm{NH}_{2} \mathrm{Cl}\right.$ and $\left.\left(\mathrm{NH}_{4}\right)_{2} \mathrm{SO}_{4}\right]$.

For a stationary microdroplet trapped in the SVELT null point its weight $m g$ is balanced by the opposing electrostatic force $q V_{\mathrm{dc}}$ :

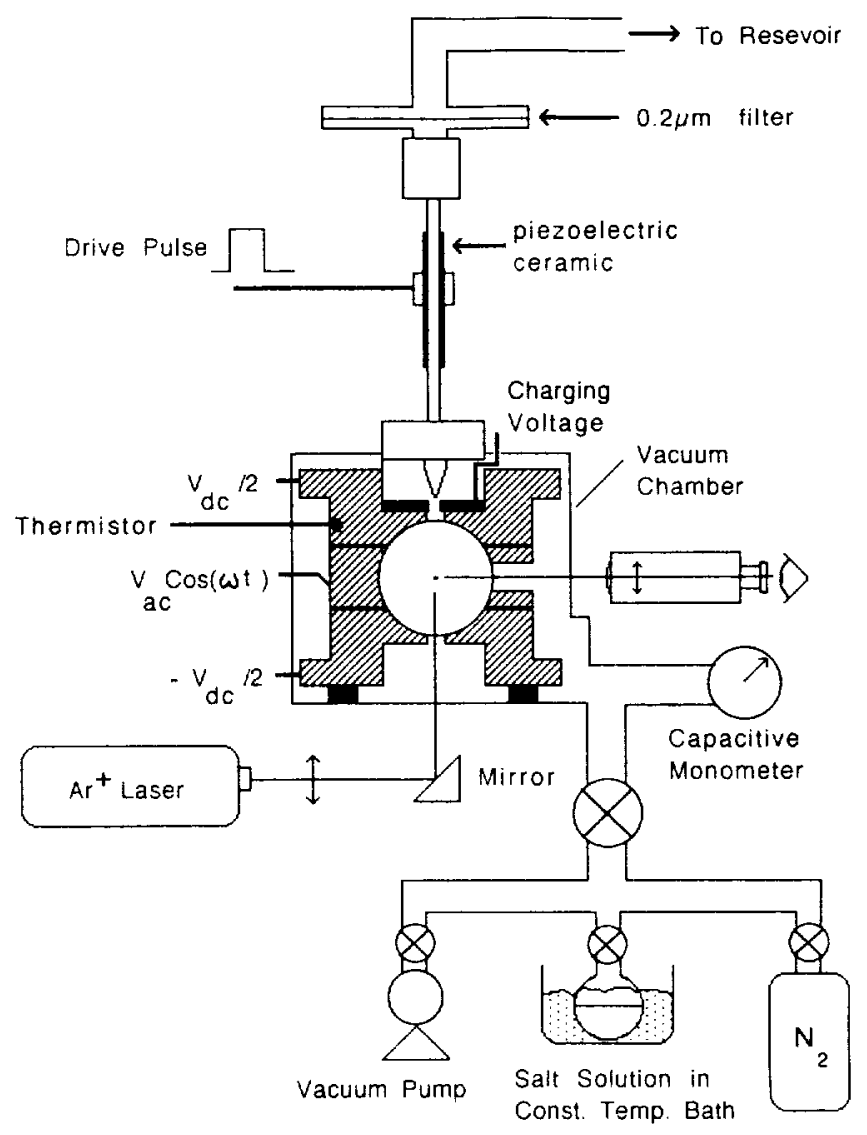

FIG. 1. Schematic drawing of the SVELT experimental apparatus.

$$
m g=C \frac{q V_{\mathrm{dc}}}{z_{0}},
$$

where $q$ is the microdroplet electrical charge, $2 z_{0}$ is the distance between the SVELT spheroid electrodes, and $C$ is the SVELT geometrical constant. Thus, by means of this equation and under an assumption that the microdroplet charge remains unaltered during an experiment the relative microdroplet mass changes can be easily determined by measuring the balancing dc voltage $V_{\mathrm{dc}}$ required to balance the weight $m g$ of the charged solution microdroplet. The assumption of the constant microdroplet charge $q$ can be easily verified in each experiment. This allows us to express the microdroplet solute concentration $n_{0}$ as follows:

$$
n_{0}=\frac{1000}{\left(M_{W}\right)_{\mathrm{slv}}}\left(\frac{V_{\mathrm{dc}}^{\mathrm{wet}}}{V_{\mathrm{dc}}^{\mathrm{dry}}}-1\right)^{-1} \text { (in molal units), }
$$

where $\left(M_{W}\right)_{\text {slv }}$ is the solvent molecular weight $\left[\left(M_{W}\right)_{\text {water }}=18\right]$, and $V_{\mathrm{dc}}^{\text {wet }}$ and $V_{\mathrm{dc}}^{\mathrm{dry}}$ are the balancing voltages for the solution (wet) and anhydrous (dry) microdroplets, respectively.

The two, experimentally justified, assumptions that (i) solute is nonvolatile, and (ii) the solution microdroplet is in equilibrium with its vapor, allow us to achieve the desired mean solute concentration $n_{0}$ inside of the solu- 
tion microdroplet by adjusting the humidity of the SVELT chamber vapor. Assumption (ii) means that the chemical potentials $\mu_{\mathrm{slv}}^{L}\left(T, n_{0}\right)$ and $\mu_{\mathrm{slv}}^{G}\left(T, n_{0}\right)$ of the solvent in the liquid (solution) and gaseous (vapor) phases are equal. Therefore, the corresponding activities $\lambda_{\mathrm{slv}}^{L}\left(T, n_{0}\right)=\lambda_{\mathrm{slv}}^{L}(T, 0) \exp \left[\mu_{\mathrm{slv}}^{L}\left(T, n_{0}\right) / k T\right]$ and $\lambda_{\mathrm{slv}}^{G}\left(T, n_{00}\right)$ $=\lambda_{\text {slv }}^{G}(T, 0) \exp \left[\mu_{\text {slv }}^{G}\left(T, n_{0}\right) / k T\right]$ are also equal. Assuming that solvent vapor can be considered as an ideal gas it is straightforward to demonstrate that [22]

$$
\begin{aligned}
\frac{\lambda_{\mathrm{slv}}^{L}\left(T, n_{0}\right)}{\lambda_{\mathrm{slv}}^{L}(T, 0)} & =\frac{\lambda_{\mathrm{slv}}^{G}\left(T, n_{0}\right)}{\lambda_{\mathrm{slv}}^{G}(T, 0)}=\frac{P\left(T, n_{0}\right)}{P(T, 0)} \\
& =\frac{P\left(T, n_{0}\right)}{P_{\text {sat }}(T)}=R_{H}\left(T, n_{0}\right),
\end{aligned}
$$

where $P\left(T, n_{0}\right)$ and $R_{H}\left(T, n_{0}\right)$ are the pressure and the relative humidity, respectively, of the solvent vapor which is in equilibrium with the solution microdroplet of the mean solute concentration $n_{0}$ and $P(T, 0)=P_{\text {sat }}(T)$ is the pressure of solvent vapor which is in equilibrium with the pure liquid solvent [this pressure is the solvent vapor saturation pressure $P_{\text {sat }}(T)$ at the given temperature $T$ ]. In reality, the solution solvent activity is dependent on the microdroplet surface curvature and charge. However, these effects are known to be insignificant for the size of the microdroplets being suspended [14].

The mean solute concentration $n_{0}$ and the corresponding solution water activity $\lambda_{\text {slv }}^{L}\left(T, n_{0}\right)$ can be easily determined by measuring the balancing dc voltage $V_{\mathrm{dc}}$ and the chamber vapor pressure. Once a microdroplet is caught and centered at the SVELT null point, the chamber can be evacuated. After the voltage $V_{\mathrm{dc}}$ of the dry microparticle is recorded, solvent vapor above the vapor reservoir is allowed to bleed back into the chamber until the solid microparticle is transformed into solution microdroplet. The second evacuation is then commenced at a slower rate by adjusting the needle value. This procedure increases the solute concentration $n_{0}$ and thus leads to deeper penetration into the supersaturated (metastability) region. Evacuation is continued at the slower rate until the crystallization point is reached. At that chamber pressure the balancing dc voltage $V_{\mathrm{dc}}$ drops precipitously. After crystallization the evacuation is continued to ensure that there has been no charge loss during the cycle. Therefore, the described experimental procedure allows continuous recording of the chamber pressure $P\left(T, n_{0}\right)$ and the voltage $V_{\mathrm{dc}}$ with the microdroplet at the SVELT geometric center.

As a result of the solvent vapor evaporation, the balancing dc voltage $V_{\mathrm{dc}}$ decreases steadily and the solution microdroplet becomes supersaturated and eventually crystallizes. This measurement is repeated several (usually three) times to ensure the reproducibility for the given solution microdroplet.

In our experimental study we have investigated the SVELT confined microdroplets of aqueous electrolyte solutions of sodium bromide $(\mathrm{NaBr})$, potassium chloride and bromide $(\mathrm{KCl}$ and $\mathrm{KBr}$ ), and ammonium chloride and sulfate $\left[\mathrm{NH}_{2} \mathrm{Cl}\right.$ and $\left.\left(\mathrm{NH}_{4}\right)_{2} \mathrm{SO}_{4}\right]$ at the temperature $T=298 \mathrm{~K}$. The relative humidity $R_{H}\left(T, n_{0}\right)$ has been measured versus the mean solute concentration $n_{0}$ in the wide range $\Delta n=n_{0}-n_{\text {sat }}$ corresponding to the high solution supersaturation $\left(n_{0} \geq n_{\text {sat }}\right.$, i.e., to the deep solution metastable states). Experiments with all solutions were performed three times under the same conditions in order to guarantee reproducibility. It has been observed that the standard deviation $\sigma_{R_{H}}\left(T, n_{0}\right)$ of the relative humidity $R_{H}\left(T, n_{0}\right)$ is a growing function of $n_{0}$. Deep penetration into the zone of metastable states was accompanied by an increase of $\sigma_{\mathrm{RH}}\left(T, n_{0}\right)$. This behavior of the standard deviation $\sigma_{\mathrm{RH}}\left(T, n_{0}\right)$ at high supersaturations is a result of local fluctuations of solute concentration and is discussed in [23]. The increase is conditioned by the corresponding increase of fluctuations of the local solute concentration $n(\mathbf{r})$ with approach to the region of unstable states (to spinodal line). Therefore, we have retained only the $R_{H}\left(T, n_{0}\right)$ data where the standard deviation is within $5 \%$.

\section{RESULTS AND CONCLUSIONS}

Figures 2 and 3 show the averaged (over three trials) experimental data for the relative vapor humidity $R_{H}\left(T, n_{0}\right)$ (square points) versus the solute concentration $n_{0}$ obtained in the SVELT experiment with aqueous microdroplets of the ammonium sulfate and ammonium chloride. Theoretical treatment (solid line) of these data is based on our results presented in Sec. III. According to result (21b) for $d \mu_{\text {slt }}\left(T, n_{0}\right) / d n_{0}$ and relation (30) for $R_{H}\left(T, n_{0}\right)$ there exists the following expression for $R_{H}\left(T, n_{0}\right)$ :

$$
R_{H}\left(T, n_{0}\right)=e^{-\left(M_{W} / 1000\right) n_{0} \psi\left(T, n_{0}\right)}
$$

where

$$
\begin{aligned}
\psi\left(T, n_{0}\right)= & 1+\frac{2^{1 / 2}(|z| e)^{3}}{48 \pi(k T)^{3 / 2}\left(\varepsilon_{0} \varepsilon\right)^{2}}\left(-\frac{\left(M_{W}\right)_{\text {slv }}}{1000} N n_{0}\right)^{1 / 2} \\
& -\frac{(|z| e)^{8} \eta}{1280 \pi^{3}(k T)^{5}\left(\varepsilon_{0} \varepsilon\right)^{7} a}\left(\frac{\left(M_{W}\right)_{\mathrm{slv}}}{1000} N n_{0}\right) .
\end{aligned}
$$

In the above expression $\psi\left(T, n_{0}\right)$ is the solvent osmotic coefficient and the solute concentration $n_{0}$ is given in molal units $\left[n_{0}\right.$ (in number density units $)=\left(M_{W}\right)_{s l v}$ $\times n_{0}$ (in molal units) $/ 1000$ ]. The experimental data for the relative humidity at the temperature $298 \mathrm{~K}$ are extrapolated by means of expression (31) in which the solvent osmotic coefficient $\psi\left(n_{0}\right)$ assumes the following form:

$$
\psi\left(T, n_{0}\right)=1+c_{1}(T) n_{0}^{1 / 2}-c_{2}(T) n_{0},
$$

where

$$
\begin{aligned}
& c_{1}(T)=\frac{2^{1 / 2}(|z| e)^{3}}{48 \pi(k T)^{3 / 2}\left(\varepsilon_{0} \varepsilon\right)^{2}}\left(\frac{\left(M_{W}\right)_{\mathrm{slv}}}{1000} N\right)^{1 / 2}, \\
& c_{2}(T)=-\frac{(|z| e)^{8} \eta}{1280 \pi^{3}(k T)^{5}\left(\varepsilon_{0} \varepsilon\right)^{7} a}\left(\frac{\left(M_{W}\right)_{\mathrm{slv}}}{1000} N\right) .
\end{aligned}
$$

The numerical expressions for the temperature-dependent coefficients $c_{1}(T)$ and $c_{2}(T)$ can be found by the least- 


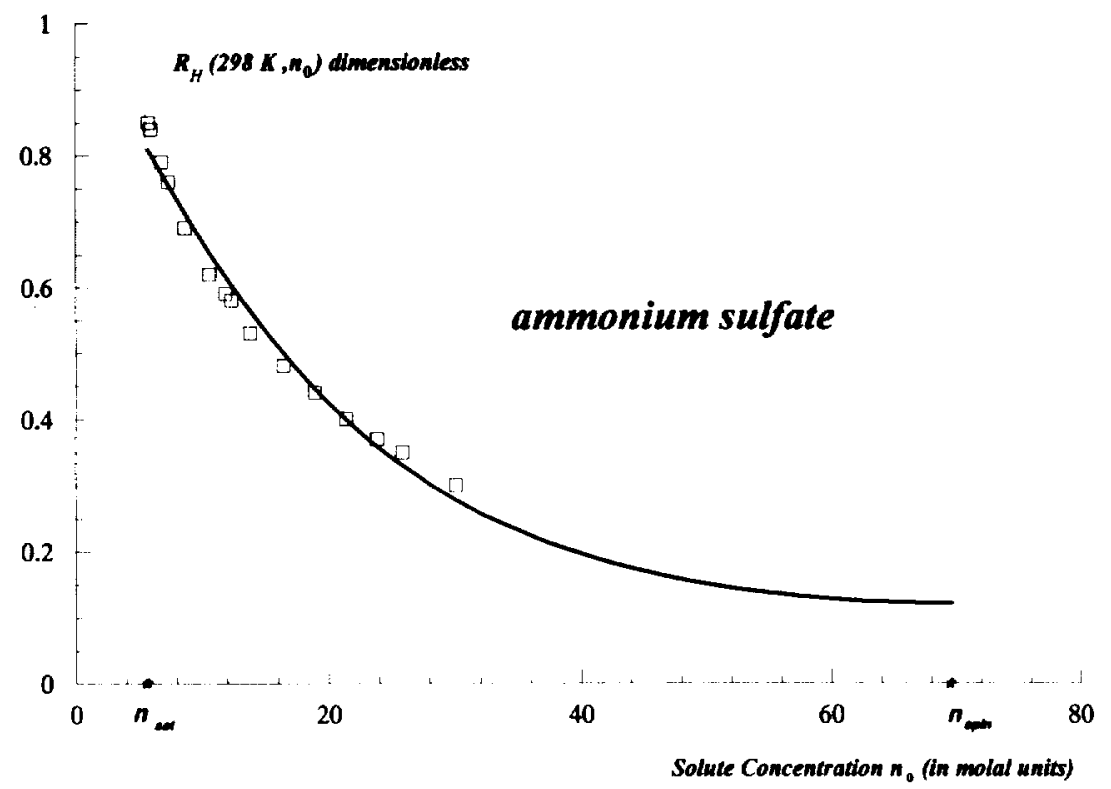

FIG. 2. Dependence of the relative humidity $R_{H}\left(T, n_{0}\right)$ of the chamber solvent vapor on the mean solute concentration $n_{0}$ of the SVELT confined solution microdroplet. The $R_{H}\left(T, n_{0}\right)$ measurements correspond to $T=298 \mathrm{~K}$. Square points represent experimental data standard deviation which is within $5 \%$ for each $n_{0}$ value. The solid line is the theoretical restoration of the obtained experimental data for supersaturated aqueous solution of ammonium sulfate.

square method applied for the experimentally obtained data for $R_{H}\left(T, n_{0}\right)$ versus $n_{0}$. These constants for each system are presented in Table $I$. The error $\Delta(T=298 \mathrm{~K})$ of the experimental data $R_{H}\left(T, n_{0}\right)$ restoration with the help of expressions (31) and (32) is very small (within 5\%) for all the solutions investigated (see Table I). Figures 2 and 3, for example, give comparison between experiment (square points) and theoretical (solid line) restoration by means of the results of (31) and (32) for microdroplets of supersaturated aqueous solutions of ammonium sulfate and ammonium chloride, respectively. In these cases the errors $\Delta_{\text {ammon sulf }}(T=298 \mathrm{~K})=4.2929 \%$ and $\Delta_{\text {ammon chlor }}$ $(T=298 \mathrm{~K})=1.4568 \%$.

The analytical expressions (32) obtained for the experimentally measured quantities $c_{1}(T)$ and $c_{2}(T)$ allow the rewriting of Eq. (23) for the spinodal concentration

$$
\begin{aligned}
& n_{\text {spin }}(T) \text { : } \\
& \quad 1+\frac{3}{2} c_{1}(T) n_{\text {spin }}^{1 / 2}(T)-\frac{1}{2} c_{2}(T) n_{\text {spin }}(T)=0 .
\end{aligned}
$$

Solution of this equation is given by the expression:

$$
\begin{aligned}
n_{\text {spin }}(T)= & 2+\frac{9}{2}\left(\frac{c_{1}(T)}{c_{2}(T)}\right]^{2} \\
& +3 \frac{c_{1}(T)}{c_{2}(T)}\left[2+\frac{9}{4}\left[\frac{c_{1}(T)}{c_{2}(T)}\right]^{2}\right]^{1 / 2}
\end{aligned}
$$

Numerical results for the spinodal concentrations $n_{\text {spin }}(T)$ at $T=298 \mathrm{~K}$ for various solutions are given in Table I. It is noteworthy that in the SVELT performed experiments with suspended microdroplets we could reach almost spinodal concentration for the supersaturat-

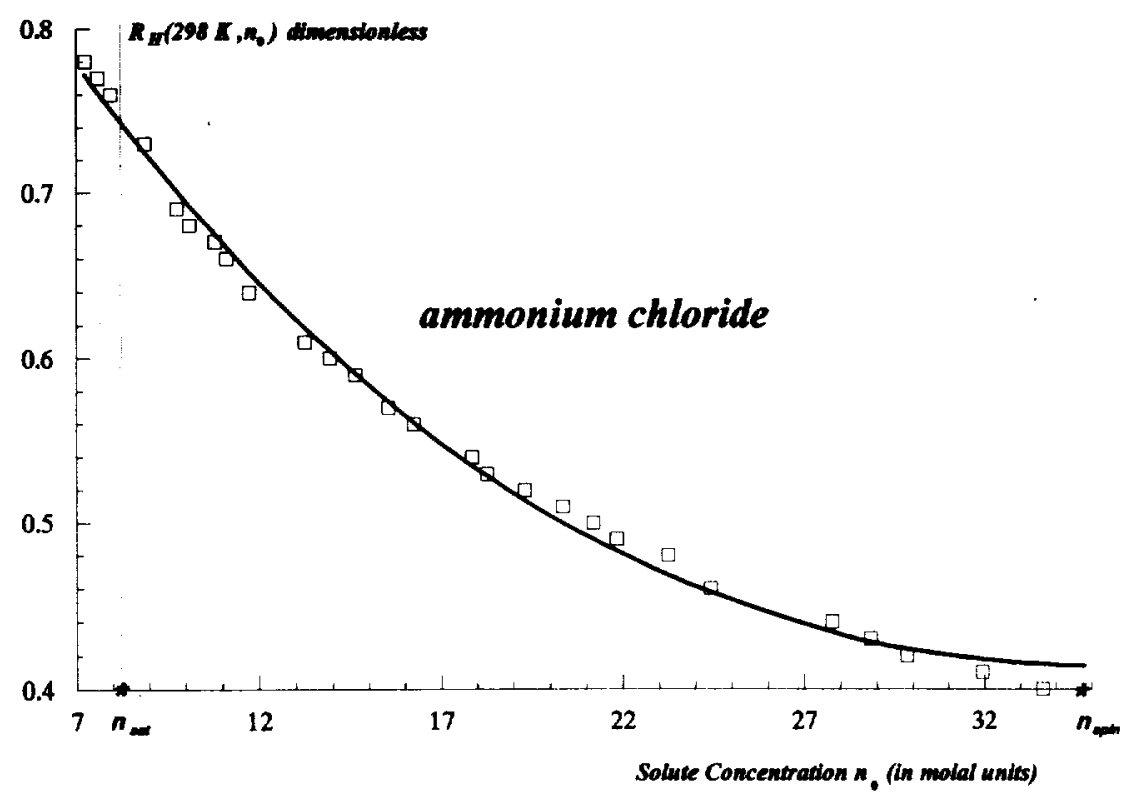

FIG. 3. Dependence of the relative humidity $R_{H}\left(T, n_{0}\right)$ of the chamber solvent vapor on the mean solute concentration $n_{0}$ of the SVELT confined solution microdroplet. The $R_{H}\left(T, n_{0}\right)$ measurements correspond to $T=298 \mathrm{~K}$. Square points represent experimental data standard deviation which is within $5 \%$ for each $n_{0}$ value. The solid line is the theoretical restoration of the obtained experimental data for supersaturated aqueous solution of ammonium chloride. 
TABLE I. $\left(M_{W}\right)_{\text {slt }}$ is the solute molecular weight, the concentrations $n_{\text {sat }}, n_{\text {spin }}$, and $n_{0, \max }$ are in molal units. All data correspond to and measurements are obtained at $T=298 \mathrm{~K}$.

\begin{tabular}{llrlrrrrrr}
\hline No. & Solute & $\left(M_{H}\right)_{\text {slt }}$ & $z$ & $n_{\text {sat }}$ & \multicolumn{1}{c}{$n_{\text {spin }}$} & $n_{0, \max }$ & $c_{1}$ & $c_{2}$ & $\Delta(\%)$ \\
\hline 1 & $\mathrm{NaBr}$ & 103 & 2 & 9.1835 & 62.4646 & 34.7655 & 1.1660 & 0.1187 & 2.5267 \\
2 & $\mathrm{KCl}$ & 75 & 1 & 4.7826 & 122.5536 & 63.9105 & 0.5586 & 0.0419 & 1.7180 \\
3 & $\mathrm{KBr}$ & 116 & 1 & 5.6962 & 66.2428 & 34.3240 & 0.7355 & 0.0753 & 0.9711 \\
4 & $\mathrm{NH}_{2} \mathrm{Cl}$ & 54 & 1 & 7.2733 & 34.7944 & 15.8927 & 0.6152 & 0.0926 & 1.4568 \\
5 & $\left(\mathrm{NH}_{4}\right)_{2} \mathrm{SO}_{4}$ & 132 & 1 & 5.7854 & 69.6309 & 34.2379 & 0.5671 & 0.0581 & 4.2939 \\
\hline \hline
\end{tabular}

ed aqueous solution of ammonium chloride. For this solution the averaged nucleation event occurred at $n(T=298 \mathrm{~K})=33.64$ (in molal units) whereas the analytically derived result for $n_{\text {spin }}(T=298 \mathrm{~K})=34.79$. Therefore, the experimental data obtained for supersaturated aqueous solutions and its treatment within the theoretical approach have provided a unique opportunity to calculate spinodal concentrations at any temperature and, thus, to calculate zones of metastable states.

In Sec. $V$ we obtained the result that within a region of the electrolyte solution metastable states there is a particular concentration $n_{0, \max }(T)$ when the supersaturated electrolyte solution experiences a dramatic decrease to zero of its electrical conductivity. At this concentration the solution becomes nonconductive since all ions become associated into electrically neutral Bjerrum's pairs and their associations. Further penetration into metastable zone leads back to the appearance of free ions and, thus, to nonzero conductivity. Expression (27) for the concentration $n_{0, \max }(T)$ can be rewritten in terms of the experimentally obtainable coefficients $c_{1}(T)$ and $c_{2}(T)$, given by relations (32), as follows:

$$
n_{0, \max }(T)=\left(\frac{3 c_{1}(T)}{5 c_{2}(T)}\right)^{2} .
$$

Numerical results for the concentrations $n_{0, \max }(T)$ at $T=298 \mathrm{~K}$ for various solutions are given in Table $\mathrm{I}$. The theoretical effect of zero conductivity of the supersaturate electrolyte solutions at the concentration $n_{0, \max }(T)$ can be independently verified in the SVELT experiments with confined solution microdroplets [24]. We are planning to perform these experiments in the near future. The calculated association degree $d\left(T, n_{0}\right)$ as a function of the solute concentration $n_{0}$ at $T=298 \mathrm{~K}$ is given in Fig. 4 for aqueous supersaturated ammonium sulfate solution.

The formalism developed in this paper allows the expression of the Landau parameters $\mu$ and $\eta$ of the $\omega$ subsystem free energy in terms of the experimentally measured coefficients $c_{1}(T)$ and $c_{2}(T)$ :

$$
\mu=-|k T|, \quad \eta=\frac{10 \pi c u_{2}(T)\left(\varepsilon_{0} \varepsilon\right)^{3}(k T)^{2}}{9 c_{1}^{2}(T)(|z| e)^{2}} a,
$$

where coefficients $c_{1}(T)$ and $c_{2}(T)$ are given by expressions (32). With the help of this result one can justify the assumption employed in Sec. II that $e^{2} \kappa_{2} \ll 1$ :

$$
\begin{aligned}
& \left(e^{2} \kappa_{2}\right)_{\max }=\Lambda(T) a, \\
& \Lambda(T)=\frac{2560 \pi^{3} c_{2}(T)\left(\varepsilon_{0} \varepsilon\right)^{3} k T n_{\text {spin }}(T)}{|z|^{4} e^{2}} .
\end{aligned}
$$

Calculation of the coefficient $\Lambda(T)$ results in very small values for all systems studied. For example, $\Lambda_{\text {ammon sulf }}(T) \approx 10^{-6}$.

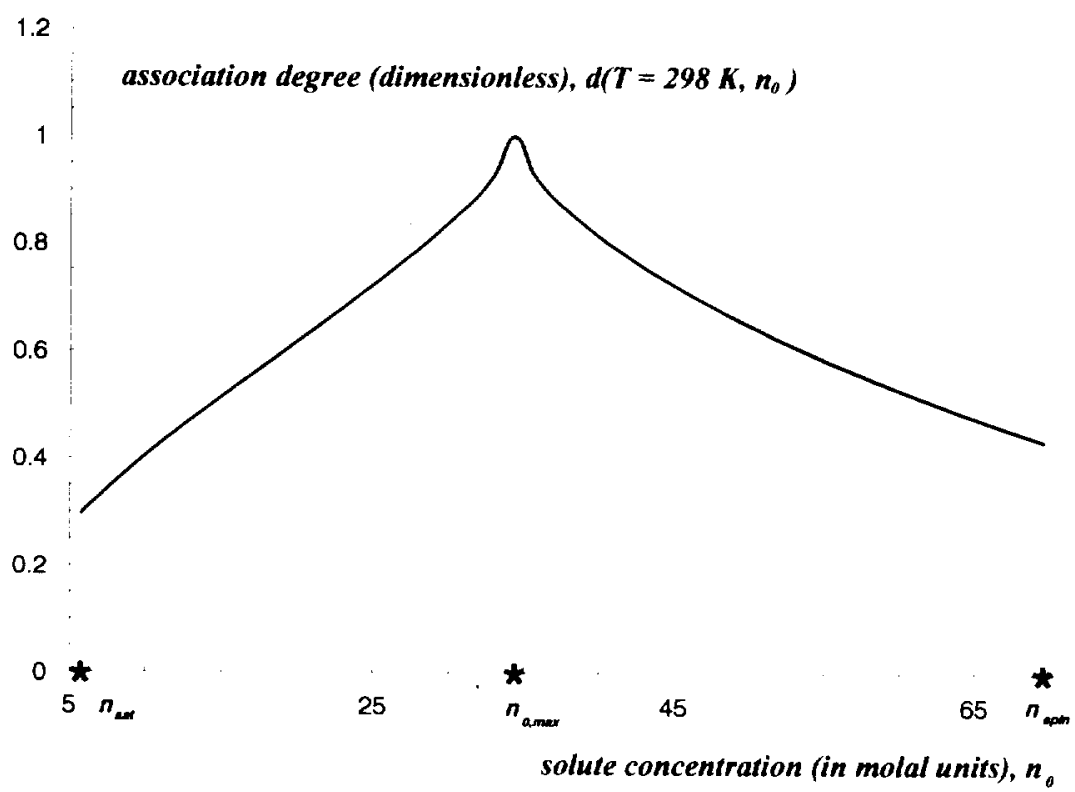


Phase separation (nucleation) results in the formation of the new stable solute-rich phase in the form of critical solute nuclei (clusters). In general, these clusters are aggregates of interacting monomers (nucleation monomers) which can be treated as elementary units appeared in the result of phase separation. The critical clusters have a quite complicated internal structure investigation of which requires a separate study. However, estimation of the number size of nucleation monomers which we call the critical number size $N_{c}\left(T, n_{0}\right)$ can be carried out within the approach suggested in this paper. Since the direct calculation of the finite sum in expression (29) is impossible, it is of use to estimate its lower $N_{c, \min }\left(T, n_{0}\right)$ and upper $N_{c, \max }\left(T, n_{0}\right)$ limits of the critical number size $N_{c}\left(T, n_{0}\right)$. To perform these estimations we introduce the following natural constraints imposed on $N_{c}\left(T, n_{0}\right)$ :

$$
N_{c}\left(T, n_{\text {sat }}(T)\right)=\infty, N_{c}\left(T, n_{\text {spin }}(T)\right)=1 .
$$

The meaning of these constraints is straightforward: the infinitely retarded nucleation at the saturation concentra- tion $n_{\text {sat }}(T)$ requires spontaneous birth of the nucleation monomers of infinite number size, whereas at the spinodal concentration $n_{\text {spin }}(T)$, where phase separation occurs instantaneously, the nucleation monomer number size is equal to 1 . The last statement means that at the spinodal concentration $n_{\text {spin }}(T)$ the monomers of the new soluterich phase are rather single Bjerrum pairs than their aggregates. It is well known that at the spinodal concentration these monomers (in our case single Bjerrum's pairs) form infinite critical clusters $[8,25]$. The structure of these clusters was substantiated to the ramified (fractal) [25]. Estimation of $N_{c, \text { min }}\left(T, n_{0}\right)$ implies that the equilibrium bulk energy $\beta(T) l$ of nucleation monomer is much greater than its surface energy $\alpha(T) l^{2 / 3}$, i.e., $\beta(T) l^{1 / 3} \gg \alpha(T)$, whereas estimation of $N_{c, \max }\left(T, n_{0}\right)$ requires an assumption that these energies are of the same order: $\beta(T) l^{1 / 3} \approx \alpha(T)$. Therefore, valuation of relation (29) under the above constraints allows us to find expressions for the lower and upper limits of the critical number size $N_{c}\left(T, n_{0}\right)$ in the forms

$$
\begin{aligned}
& N_{c, \min }\left(T, n_{0}\right)=\frac{1}{\ln \left[\frac{n_{0}}{n_{\mathrm{sat}}(T)}\right]} \ln \left[1-\frac{n_{\mathrm{spin}}(T)}{d\left(T, n_{\mathrm{spin}}(T)\right)} \frac{d\left(T, n_{0}\right)}{n_{0}}\left(1-\frac{n_{0}}{n_{\mathrm{sat}}(T)}\right)\right], \\
& N_{c, \max }\left(T, n_{0}\right)=\frac{1}{\ln \left[\xi(T) n_{0}\right]} \ln \left[1-\frac{n_{\mathrm{spin}}(T)}{d\left(T, n_{\mathrm{spin}}(T)\right)} \frac{d\left(T, n_{0}\right)}{n_{0}}\left[1-\xi(T) n_{0}\right]\right),
\end{aligned}
$$

where

$$
\xi(T)=\frac{1}{n_{\mathrm{sat}}(T)}-\frac{1}{n_{\mathrm{spin}}(T)} \frac{d\left(T, n_{\mathrm{spin}}(T)\right)}{d\left(T, n_{\mathrm{sat}}(T)\right)} .
$$

In the above expressions $n_{\text {sat }}(T)$ is the solute concentration at saturation (see Table I). The obtained experimental results at $T=298 \mathrm{~K}$ allow us to investigate how the lower $N_{c, \min }\left(n_{0}\right)=N_{c, \min }\left(T=298 \mathrm{~K}, n_{0}\right)$ and upper $N_{c, \max }\left(n_{0}\right)=N_{c, \max }\left(T=298 \mathrm{~K}, n_{0}\right)$ limits of the critical number size $N_{c}\left(n_{0}\right)$ depend on the solute concentration $n_{0}$. This requires substitution into expressions (36a) and (36b) of result (34) for $n_{\text {spin }}(T)$ and table values for $n_{\text {sat }}(T)$ as well as relation (26) calculated for the given $n_{\text {spin }}(T)$ and $n_{\text {sat }}(T)$. Figure 5 gives the dependence of $N_{c, \min }\left(n_{0}\right)$ and $N_{c, \max }\left(n_{0}\right)$ on $n_{0}$ in the range $\left[n_{\text {spin }}, n_{\text {sat }}\right]$ for the aqueous supersaturated electrolyte solution of ammonium sulfate. In Table II there are values for $N_{c, \min }\left(n_{0}\right)$ and $N_{c, \max }\left(n_{0}\right)$ at the solute concentrations
$1.1 \times n_{\text {sat }}$ and $n_{\text {spin }}$ for various solutions. Analysis of Fig. 5 together with the analogous figures for the other supersaturated solutions studied shows that for all the cases the critical number size $N_{c}\left(n_{0}\right)$ is a decreasing function of solute concentration $n_{0}$ with the following properties: (i) $N_{c}\left(n_{\text {sat }}(T)\right)=\infty$, (ii) $N_{c}\left(n_{\text {sp }}(T)\right)=1$, and (iii) $N_{c}\left(T, n_{0}\right)$ has a local maximum at the concentration $n_{0}=n_{0, \max }(T)$. Existence of this local maximum can be understood if one takes into account that at the concentration $n_{0, \max }(T)$ all ions become associated into the electrically neutral Bjerrum pairs and electrolyte solution becomes nonelectrolyte. This leads to a dramatic change of the solution physicochemical properties, such as its electrical conductivity, at the solute concentration $n_{0, \max }(T)$.

The estimations performed of the lower $N_{c, \min }\left(T, n_{0}\right)$ and upper $N_{c, \max }\left(T, n_{0}\right)$ limits of the nucleation critical number size $N_{c}\left(T, n_{0}\right)$ allow determination of simple analytical expressions for the surface tension $\alpha(T)$ and the equilibrium bulk energy $\beta(T)$ per solute molecule:

TABLE II. In this table all calculated quantities are obtained at $T=298 \mathrm{~K}$.

\begin{tabular}{llcccccc}
\hline \hline No. & \multicolumn{1}{c}{ Solute } & $\alpha$ & $\beta$ & $N_{c, \min }\left(n_{\text {sat }}+0.1\right)$ & $N_{c, \min }\left(n_{\text {spin }}\right)$ & $N_{c, \max }\left(n_{\text {sat }}+0.1\right)$ & $N_{c, \max }\left(n_{\text {spin }}\right)$ \\
\hline 1 & $\mathrm{NaBr}$ & 7.0206 & 0.2198 & 4.9374 & 1 & 14.4593 & 1 \\
2 & $\mathrm{KCl}$ & 7.1265 & 0.1007 & 9.4578 & 1 & 19.8006 & 1 \\
3 & $\mathrm{KBr}$ & 6.6947 & 0.1466 & 6.9080 & 1 & 16.5334 & 1 \\
4 & $\mathrm{NH}_{2} \mathrm{Cl}$ & 6.4823 & 0.1662 & 6.2668 & 1 & 16.4945 & 1 \\
5 & $\left(\mathrm{NH}_{4}\right)_{2} \mathrm{SO}_{4}$ & 6.8338 & 0.1292 & 8.1944 & 1 & 33.8936 & 1 \\
\hline \hline
\end{tabular}




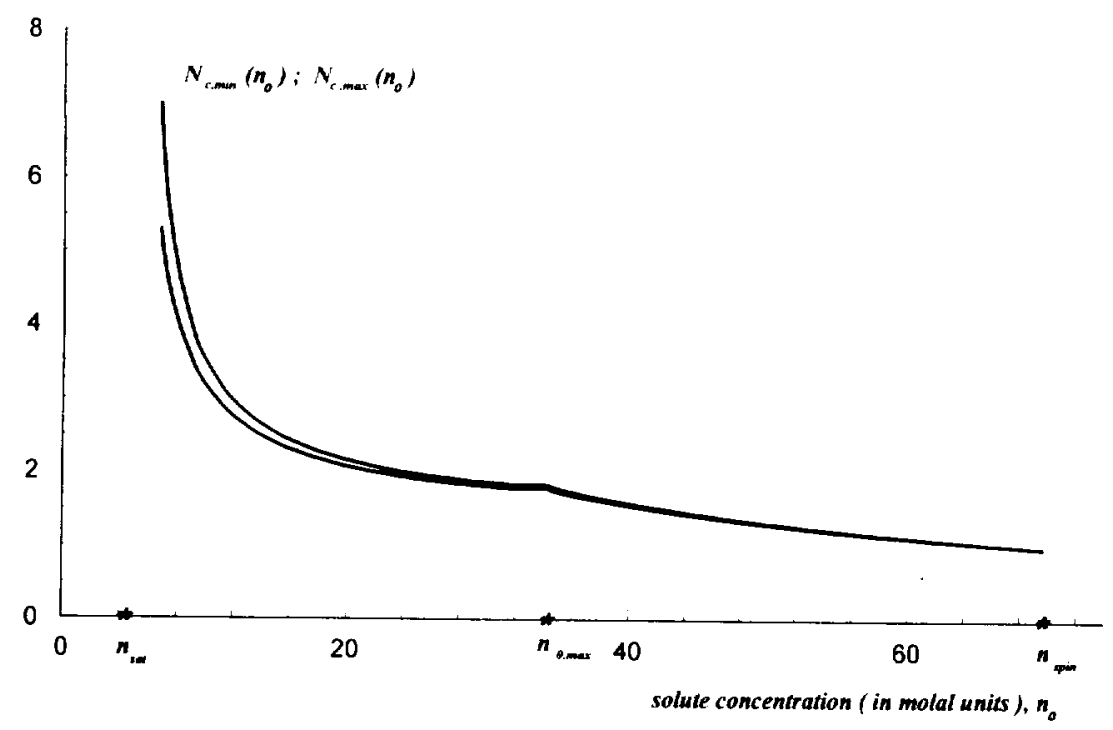

FIG. 5. Dependence of the lower $N_{c, \min }\left(T, n_{0}\right)$ and upper $N_{c, \max }\left(T, n_{0}\right)$ limits of the critical number size $N_{c}\left(T, n_{0}\right)$ on the mean solute concentration $n_{0}$ at $T=298 \mathrm{~K}$ for supersaturated aqueous solution of ammonium sulfate.

$\alpha(T)=-\ln \left[n_{\mathrm{sat}}(T)\right], \quad \beta(T)=-\ln \left[\xi(T) n_{\mathrm{sat}}(T)\right]$,

where all concentrations are given in molal units. The calculated values for $\alpha(T)$ and $\beta(T)$ at $T=298 \mathrm{~K}$ for the investigated supersaturated solutions can be found in Table II.

The conclusions presented above summarize that combination of the high precision SVELT experiments with the theory developed for supersaturated electrolyte solutions has allowed us to obtain a number of interesting re- sults in the physics of supersaturated electrolyte solutions.

\section{ACKNOWLEDGMENTS}

The authors gratefully acknowledge the support of the National Science Foundation (NSF Grant No. CTS 9020233) and NASA (NASA Grants No. NA68-960 and No. NAG8-1060). The authors thank S. Arnold for his help in creating the experiment apparatus and useful remarks related to this work.
[1] P. Debye and E. Hückel, Phys. Z. 24, 185 (1923).

[2] Nucleation Phenomena, edited by A. C. Zettlemoyer (Elsevier, Amsterdam, 1977).

[3] J. D. Gunton, M. San Miguel, and P. S. Sahni, in Phase Transitions and Critical Phenomena, edited by C. Domb and J. Lebowitz (Academic, New York, 1983), Vol. VIII.

[4] A. S. Myerson and A. F. Izmailov, in Handbook of Crystal Growth, edited by D. T. J. Hurle (Elsevier, Amsterdam, 1993), Vol. la.

[5] L. D. Landau and E. M. Lifshitz, Statistical Physics (Pergamon, London, 1986), Vol. 5.

[6] H. Metiu, K. Kitahara, and J. Ross, in Studies in Statistical Mechanics, edited by E. W. Montroll and J. L. Lebowitz (North-Holland, Amsterdam, 1979), Vol. VII.

[7] V. L. Ginzburg and L. D. Landau, in Collected Papers of L. D. Landau, edited by D. ter Haar (Pergamon, London, 1965).

[8] J. W. Cahn and J. E. Hilliard, J. Chem. Phys. 28, 258 (1958).

[9] J. W. Cahn and J. E. Hilliard, J. Chem. Phys. 31, 688 (1959)

[10] V. L. Ginzburg, Sov. Phys. Solid State Phys. 2, 2034 (1960).

[11] A. P. Levanyuk and A. A. Sobyanin, JETP Lett. 11, 371 (1970).

[12] N. Bjerrum, Kgl. Danske Vidensk.Selskab. 7, No. 9 (1926).
[13] J. Frenkel, Kinetic Theory of Liquids (Dover, New York, 1955).

[14] G. O. Rubel, J. Aerosol. Sci. 12, 551 (1981).

[15] C. B. Richardson and J. F. Spann, J. Aerosol Sci. 15, 563 (1984).

[16] N. Tang, H. R. Munkelwitz, and N. Wang, J. Aerosol Sci. 114, 409 (1986).

[17] S. Arnold and L. M. Folan, Rev. Sci. Instrum. 57, 2250 (1986).

[18] S. Arnold, Rev. Sci. Instrum. 62, 3025 (1991).

[19] A. S. Myerson, H.-S. Na, A. F. Izmailov, and S. Arnold, in Proceedings of the 12th Symposium on Industrial Crystallization, Warsaw, 1993, edited by Z. H. Rojkowski (Wlodarski, Warsaw, 1993), Vol. 2, p. 3-013.

[20] H.-S. Na, Ph.D. dissertation, Polytechnic University, 1994 (unpublished).

[21] H.-S. Na, S. Arnold and A. S. Myerson, J. Cryst. Growth 139, 104 (1994).

[22] E. A. Guggenheim, Thermodynamics. An Advanced Treatment for Chemists and Physicks (North-Holland, Amsterdam, 1967).

[23] A. S. Myerson and A. F. Izmailov, J. Phys. D 26, B123 (1993).

[24] S. Arnold (private communication).

[25] C. Unger and W. Klein, Phys. Rev. B 29, 2698 (1984). 
